\title{
Motion of spheres along a fluid-gas interface
}

\author{
Bogdan Cichocki \\ Institute of Theoretical Physics, Warsaw University, Hoża 69, 00-681 Warsaw, Poland \\ Maria L. Ekiel-Jeżewska ${ }^{\text {a) }}$ \\ Institute of Fundamental Technological Research, Polish Academy of Sciences, Świętokrzyska 21, \\ 00-049 Warsaw, Poland \\ Gerhard Nägele \\ Institut für Festkörperforschung, Forschungszentrum Jülich, D-52425 Jülich, Germany \\ Eligiusz Wajnryb \\ Institute of Fundamental Technological Research, Polish Academy of Sciences, Swiętokrzyska 21, \\ 00-049 Warsaw, Poland
}

(Received 9 February 2004; accepted 4 May 2004)

\begin{abstract}
A system of many spherical particles, suspended in a quiescent fluid and touching a planar free fluid-gas interface, is considered. Stick fluid boundary conditions at the sphere surfaces are assumed. The free surface boundary conditions are taken into account with the use of the method of images. For such a quasi-two-dimensional system, the one-sphere resistance operator is calculated numerically. Moreover, the corresponding friction and mobility tensors are constructed from irreducible multipole expansion. Finally, the long-distance terms of the two-sphere mobility tensor are evaluated explicitly up to the order of $1 / r^{3}$, where $r$ is the interparticle distance. Experiments which have motivated this work are outlined. () 2004 American Institute of Physics.
\end{abstract}

[DOI: $10.1063 / 1.1766016$ ]

\section{INTRODUCTION}

The dynamics of mesoscopically large particles suspended in a viscous fluid is strongly influenced, additionally to direct interparticle forces, by fluid-mediated hydrodynamic interactions. ${ }^{1-3}$ In the past, research was mainly focused on the microhydrodynamics of three-dimensional bulk dispersions of particles interacting through, e.g., excluded volume $^{4-6}$ or electrostatic direct forces. ${ }^{7,8}$ Meanwhile the center of interest has shifted to properties of colloids near boundaries like a single hard wall, ${ }^{9-14}$ and colloids close to a clean ${ }^{15,16}$ or surfactant-covered fluid-fluid interface. ${ }^{17}$ Of particular interest are so-called quasi-two-dimensional dispersions where the particles are confined to move essentially laterally in between two parallel walls, ${ }^{18-27}$ and along liquidgas interfaces. ${ }^{28-36}$ The presence of walls or interfaces complicates the particle dynamics since additional fluid boundary conditions have to be satisfied, with the flow field being reflected from the interface. There are remarkable effects associated with particle-wall hydrodynamic interactions like the apparent mutual attraction of two likecharged colloidal spheres which are repelled from a nearby charged wall. ${ }^{37-39}$ In addition there are fundamental theoretical problems encountered in quasi-two-dimensional systems related to the existence of certain diffusion transport coefficients, ${ }^{29,30,35}$ the possibility of two-stage continuous freezing processes with an intermediate hexatic phase, and vitrification in nonmonodisperse systems. Recent work on systems with boundary effects comprise sedimentation, ${ }^{11,40}$ adsorption, and

${ }^{a)}$ Electronic address: mekiel@ippt.gov.pl escape $^{14,41}$ of particles near a wall, the dynamics of quasitwo-dimensional monolayers of colloidal spheres between parallel walls, ${ }^{21-27}$ and at fluid-gas interfaces.

A well-studied example of the latter case is given by micron-sized super-paramagnetic colloidal spheres suspended in water next to a water-air interface. ${ }^{31-33,36}$ By means of an experimental hanging-drop geometry, the spheres are gravitationally confined to lateral motion along the interface. The surface tension of water is so large, and the buoyancy-corrected gravitational force acting downwards on the spheres so small in comparison, that the interface is practically planar in its experimentally scanned sector. The spheres repel each other through long-range dipole magnetic forces induced by an external magnetic field pointing perpendicular to the interface. This model system is of particular interest both from the theoretical and experimental point of view since the direct interactions are, as compared to other colloidal systems, very well known. Moreover, the strength of the dipole interactions can be precisely tuned by the magnetic field strength. The two-dimensional particle trajectories can be monitored over an extended range of times using video imaging. This method allows to study in great detail phenomena such as hydrodynamic enhancement of selfdiffusion and collective diffusion, ${ }^{31,32,34-36}$ melting transitions and freezing transitions via an intermediate hexatic phase of quasi-long-range orientational order, ${ }^{33}$ and vitrification in interaction-polydisperse systems. For a quantitative description of particle diffusion in this magnetic model system, it is necessary to know precisely the lateral hydrodynamic mobility tensors of the spheres in presence of the liquid-gas interface. The mobility tensors are thus an essen- 
tial input in theoretical and Brownian dynamics simulation studies of in-plane diffusion and nonequilibrium microstructure and, most likely, affect also the vitrification dynamics of dense magnetic monolayers.

These observations have motivated us to consider, in this article, the quasi-two-dimensional hydrodynamic interactions of spheres of radius $a$, confined to lateral motion parallel to a planar fluid-gas free interface (see Fig. 1). The quiescent fluid occupies the half space $z>0$, with gravity pointing into the negative $z$ direction. The height of the sphere centers is fixed to $z=a$, i.e., the spheres always touch the interface. The Newtonian fluid of shear viscosity $\eta$ is described by the stationary Stokes equation with stick boundary conditions on the sphere surfaces. The gas phase in the lower half space $z<0$ is modeled as a fluid of (essentially) zero viscosity. Therefore, the boundary conditions for the fluid at the interface $z=0$ are zero velocity component normal to the interface, and zero tangential stress. This means that the normaltangential components, $\sigma_{y z}$ and $\sigma_{x z}$, of the fluid stress tensor, $\boldsymbol{\sigma}$, vanish at the interface. Super-paramagnetic colloidal spheres at the air-water interface are torque free, since the magnetic dipole moments induced by the external magnetic field are not coupled to the sphere material. More generally, we consider also non-torque-free spheres.

In reality, the effect of a finite surface tension is not negligible in general, and the overall planar free surface may be curved to some extent in the vicinity of a touching particle. Moreover, a liquid-gas interface always shows thermally induced undulations. However, in many experiments the approximation of a planar interface in the presence of touching microspheres is a very good one. In the hanging water drop experiments, ${ }^{31-33,36,42}$ for example, the surface tension energy associated with a magnetic colloidal sphere of diameter $4.7 \mu \mathrm{m}$ and mass density of $1.7 \mathrm{~g} / \mathrm{cm}^{3}$ is many orders of magnitude larger than the thermal energy $k_{B} T$ or the buoyancy-corrected gravitational energy. ${ }^{43}$ The thermal fluctuations in the vertical position of a microsphere ${ }^{31,44}$ and the root-mean-square amplitude of capillary waves ${ }^{44-46}$ are therefore only of the order of nanometers, i.e., less than $1 \%$ of the diameter. Due to the addition of a surfactant, the surfaces of the magnetic spheres are completely wetted by water. ${ }^{31}$ Hence, there are no capillary forces active which adhere the spheres to the liquid-air interface. ${ }^{47}$ From a balance of the surface tension energy and the gravitational energy of a microsphere at the interface, it follows that the interface is practically unperturbed by the weight of the particles; the local deformation in the initially flat interface

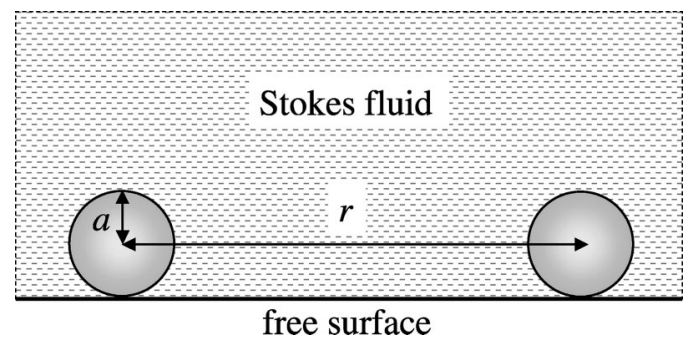

FIG. 1. Sketch of a quasi-two-dimensional system, with spheres moving within a semi-infinite fluid and touching a planar fluid-gas interface. amounts to less than $0.01 \%$ of the sphere radius. In this context it should be mentioned that Lee and Leal ${ }^{48}$ have investigated the kinematic deformation of an initially flat fluidfluid interface due to the motion of a single sphere normal to the interface. In Ref. 49, these authors provide kinematic criteria for the validity of asymptotically small interface deformations in terms of the translational and angular sphere velocities, and the shear viscosity of the embedding fluid. An application of these criteria to the hanging-drop experiments reveals again a negligibly small interface deformation in case of thermal sphere velocities.

In this paper, we totally neglect deformation of the free surface due to the surface tension and we consider $N$ spheres in a fluid bounded by a planar interface. The advantage is that with such a simplification, the algorithm to calculate the many-body hydrodynamic mobility and friction tensors for the spheres ${ }^{50}$ is based on the method of images. As in case of an unbounded fluid, the concept of induced forces ${ }^{51-54}$ and the irreducible multipole expansion ${ }^{55,56}$ are also used.

The main result of this work is the adjustment of the general approach mentioned above to the motion of $N$ spheres, which touch the interface. This problem is not straightforward. A particularly interesting theoretical conclusion which we are going to discuss is that, since the particles touch the free surface, the contact prevents them from rotating with angular velocity having a component parallel to the interface. This restriction on the allowed rotational axis holds true for all values of the external forces and torques compatible with the creeping fluid flow.

This result is not surprising if the system is interpreted as $N$ real spheres plus $N$ their images, in the unbounded fluid, extended for $z<0$ by the reflection with respect to the interface at $z=0$. In this context, each sphere touching the interface is represented by a touching doublet, i.e., the sphere plus its image. Relative motions of the touching spheres would require infinite lubrication forces or torques, ${ }^{57}$ so as they do not appear. In particular, for two touching spheres in unbounded fluid, rotation along an axis perpendicular to the line of centers is possible only as a rigid rotation of the whole doublet. However, such a rigid motion may not appear at the interface, because it would not be consistent with the symmetry of the unbounded system obtained by the method of images. (Of course, according to the discussion from the previous paragraph, it would be of interest to check experimentally to what extent the constrained-motion predictions of lubrication theory are met in real quasi-two-dimensional systems with a nonflat interface.)

The difficulty is that elimination of the "forbidden" motions deals with the infinite "constraint" lubrication forces. In this paper, to avoid a spurious divergence due to infinite lubrication forces (and also infinite higher force multipoles) of a sphere touching its image, the inherent symmetry of the system is taken into account by construction of modified multipole functions. This is an efficient method to construct the many-sphere quasi-two-dimensional mobility tensor. This tensor determines the two-dimensional (i.e., parallel to the interface) translational velocities and the one-dimensional (i.e., transverse to the interface) angular velocities of the spheres for given external two-dimensional forces and one- 
dimensional torques acting on them. Explicit results are obtained for the long-distance contributions to the self-mobility and distinct mobility tensors of two spheres up to order $1 / r^{3}$ in the interparticle distance $r$. We have already mentioned an example of a system, where these long-distance results are important. Indeed, in typical monolayers of superparamagnetic spheres the long-range and repulsive dipole interparticle forces render particle distances shorter than $r$ $<6 a$ extremely unlikely. ${ }^{31}$ Therefore the long-distance asymptotics of the translational-translational part of the quasitwo-dimensional mobility matrix of two spheres should suffice to describe the lateral translational diffusion of such torque-free spheres along the interface.

The paper is organized as follows. In Sec. II we summarize the essentials of the hydrodynamic interactions between $N$ spheres immersed in an unbounded fluid, and explain how one can calculate the many-sphere mobility and friction tensors using the multipole expansion method. In the first part of Sec. III we outline how this method has to be modified if $N$ spheres are surrounded by a semi-infinite fluid bounded by a planar free surface. In the second part, we explain the difficulties which arise when the multipole expansion method is applied to spheres which touch the interface. In the third part of Sec. III, these difficulties are resolved by a suitable reformulation of the problem, by accounting for the lubrication effects and the symmetry of the system. We derive a general expression for the quasi-two-dimensional mobility tensor of $N$ spheres moving along a planar free surface. This general expression is applied in Sec. IV to obtain the mobility of a single sphere touching a free interface. In Sec. V we derive an explicit result for the long-distance asymptotic form of the two-sphere mobility tensor, which includes all terms of linear, quadratic, and cubic order in the inverse sphere-tosphere distance $1 / r$. A final discussion of our results is given in Sec. VI.

\section{THREE-DIMENSIONAL HYDRODYNAMIC INTERACTIONS BETWEEN $\boldsymbol{N}$ SPHERES IN AN UNBOUNDED FLUID}

Let us start from a brief reminder of the multipole expansion method as a powerful tool to determine threedimensional hydrodynamic interactions between many spheres; in particular, to evaluate the friction and mobility tensors. ${ }^{1,3}$ This reminder is important, because the concepts developed in case of bulk fluids will be later applied to construct the friction and mobility tensors for quasi-twodimensional systems. In this section, we explain the method in a general context. However, in the following we will focus on the mobility tensor essential in the hydrodynamic mobility problem in which there is no incident fluid flow; the forces and torques on the spheres are given and the resulting translational and rotational velocities are seeked for. The mobility problem is basic, e.g., to diffusion of colloidal spheres as described by the generalized Smoluchowski equation.

Consider $N$ rigid spheres of equal radii $a$ immersed in an incident flow field $\mathbf{v}_{0}(\mathbf{r})$ of an incompressible and unbounded fluid of shear viscosity $\eta$. At small Reynolds numbers the fluid velocity and pressure, $\mathbf{v}(\mathbf{r})$ and $p(\mathbf{r})$, satisfy the stationary Stokes equations, ${ }^{1}$

$$
\begin{aligned}
& \boldsymbol{\nabla} \cdot \mathbf{v}=0, \\
& \eta \boldsymbol{\nabla}^{2} \mathbf{v}(\mathbf{r})-\boldsymbol{\nabla} p(\mathbf{r})=\mathbf{0},
\end{aligned}
$$

with stick boundary conditions at the particle surfaces $S_{i}$, $\mathbf{v}(\mathbf{r})=\mathbf{w}_{i}(\mathbf{r}) \equiv \mathbf{U}_{i}+\boldsymbol{\Omega}_{i} \times\left(\mathbf{r}-\mathbf{r}_{i}\right), \quad$ for $\mathbf{r} \in S_{i}, \quad i=1, \ldots, N$,

where $\mathbf{r}_{i}$ stands for the position of the center of particle $i$, while $\mathbf{U} \equiv\left(\mathbf{U}_{1}, \ldots, \mathbf{U}_{N}\right)$ and $\boldsymbol{\Omega} \equiv\left(\boldsymbol{\Omega}_{1}, \ldots, \boldsymbol{\Omega}_{N}\right)$ are the translational and the angular velocities of all the particles.

To solve Eqs. (2) and (3), the density $\mathbf{f}_{i}(\mathbf{r})$ of induced forces $^{51-54}$ is considered for each particle $i=1, \ldots, N$. These forces, located at the particle surfaces, are exerted onto the fluid by the spheres and are determined from the boundary conditions (3). The rigid body motion of the particles may be now interpreted as a fictitious fluid flow, which obeys the Stokes equations (1) and (2) for $\left|\mathbf{r}-\mathbf{r}_{i}\right| \leqslant a$. In this way Eqs. (1) and (2) augmented with the additional source term at the rhs equal to $-\sum_{i=1}^{N} \mathbf{f}_{i}(\mathbf{r})$, are valid both outside and inside the spheres. ${ }^{51-54}$ The solution of Eqs. (1) and (2) generalized in this way can then be written in terms of an integral Green operator with tensor kernel $\mathbf{T}$ (Ref. 58),

$$
\mathbf{v}(\mathbf{r})-\mathbf{v}_{0}(\mathbf{r})=\sum_{j=1}^{N} \int \mathbf{T}(\mathbf{r}, \overline{\mathbf{r}}) \mathbf{f}_{j}(\overline{\mathbf{r}}) d^{3} \overline{\mathbf{r}} .
$$

For an unbounded fluid, the integral kernel $\mathbf{T}(\mathbf{r}, \overline{\mathbf{r}})$ is equal to the Oseen tensor $\mathbf{T}_{0}(\mathbf{r}-\overline{\mathbf{r}}){ }^{1}{ }^{1}$ where

$$
\mathbf{T}_{0}(\mathbf{r})=\frac{1}{8 \pi \eta r}(\mathbf{I}+\hat{\mathbf{r}} \hat{\mathbf{r}}),
$$

and $\hat{\mathbf{r}}=\mathbf{r} / r$.

The maintask is to determine the force densities $\mathbf{f}_{i}$, $i=1, \ldots, N$. To this end, Eq. (4) is restricted to the surface $S_{i}$ of each particle $i$, and its lhs is written in terms of the stick boundary conditions (3), viz,

$$
\mathbf{w}_{i}-\mathbf{v}_{0}=\mathbf{Z}_{0}^{-1}(i) \mathbf{f}_{i}+\sum_{j \neq i}^{N} \mathbf{G}(i j) \mathbf{f}_{j}, \quad \text { for } \mathbf{r} \in S_{i},
$$

where the rhs of Eq. (4) has been decomposed into two parts. The first term at the rhs of Eq. (6) accounts for the contribution to the velocity field on $S_{i}$ from the force density located on the same surface $S_{i}$. The corresponding single-sphere integral operator is denoted as $\mathbf{Z}_{0}^{-1}(i)$ (the reason for such a notation will become clear soon). The second part is the sum of velocity fields generated by the force densities $\mathbf{f}_{j}$ of all the other particles $j \neq i$, at the surface $S_{i}$ of particle $i$. The corresponding propagators are denoted as $\mathbf{G}(i j)$. One advantage of separating off the one-particle contribution in Eq. (6) is that only $\mathbf{Z}_{0}$ must be changed when nonsticky sphere boundary conditions are used.

The above equation can be formally solved for the force densities $\mathbf{f}=\left(\mathbf{f}_{1}, \ldots, \mathbf{f}_{N}\right)$ as

$$
\mathbf{f}=\left(\mathbf{Z}_{0}^{-1}+\mathbf{G}\right)^{-1}\left(\mathbf{w}-\mathbf{v}_{0}\right) .
$$

We have used here the short-hand notation, $\mathbf{w}-\mathbf{v}_{0}$ $=\left(\mathbf{w}_{1}-\mathbf{v}_{0}, \ldots, \mathbf{w}_{N}-\mathbf{v}_{0}\right)$. The operators $\mathbf{Z}_{0}^{-1}$ and $\mathbf{G}$ invoke a summation over the particle labels $j$ according to $\sum_{j} \mathbf{Z}_{0}^{-1}(j) \delta_{i j}$ and $\Sigma_{j} \mathbf{G}(i j)\left(1-\delta_{i j}\right)$, respectively. 
In the following, we first introduce the one-particle operator $\mathbf{Z}_{0}(i)$, justifying the notation used in Eq. (6), and we explain how it is calculated. Next, we interpret the formal expression (7) for the induced force densities as the result of all subsequent velocity field reflections, ${ }^{1}$ or speaking differently, as the sum of a multiple scattering series. ${ }^{56}$ Then weexplain how $\mathbf{f}$ is calculated from Eq. (7) by means of the multipole expansion.

Consider first a system, consisting of a single sphere $i$ immersed in an ambient fluid flow field $\mathbf{v}_{0}$ and moving rigidly with a given velocity field $\mathbf{w}_{i}$. According to Eq. (7), the force density $\mathbf{f}_{i}$ of a single sphere system is formally given by the one-sphere resistance operator $\mathbf{Z}_{0}(i)$,

$$
\mathbf{f}_{i}=\mathbf{Z}_{0}(i)\left(\mathbf{w}_{i}-\mathbf{v}_{0}\right),
$$

which explains the notation used in Eq. (6). In Ref. 59, the force density $\mathbf{f}_{i}$ induced on the sphere surface has been related to the fluid flow, known from Refs. 55, 60, and 61, to determine the operator $\mathbf{Z}_{0}(i)$ explicitly. To this goal a complete set of irreducible spherical multipole functions $\mid i \operatorname{lm} \sigma)$ has been introduced. The label $i$ denotes that arguments of the functions are the relative positions measured with respect to the center of sphere $i$. The three multipole indices $l, m$, and $\sigma$ are equal to $l=1,2, \ldots, m=-l, \ldots,+l$, and $\sigma$ $=0,1,2$. The multipoles with $\sigma=0$ and $\sigma=1$ are not related to the pressure gradient; those with $\sigma=0$ are rotationless vectors while those with $\sigma=1$ are pseudovectors of nonzero vorticity. The vector multipoles with $\sigma=2$ are the only ones coupled to the pressure gradient, with nonvanishing vorticity. In addition, the set of basic functions is chosen in such a way that each multipole matrix element of the operator $\mathbf{G}(i j)$ is proportional to a power of the distance between particle $i$ and particle $j$. The irreducible multipole functions $\mid i \operatorname{lm} \sigma)$ will be used in this paper. However, our normalization differs from that used in Ref. 59. We follow a more convenient normalization introduced in Ref. 50. The force multipoles $\left(\mathbf{f}_{i} \mid i l m \sigma\right)$ have been expressed ${ }^{59}$ by the velocity multipoles $\left(\mathbf{w}_{i}-\mathbf{v}_{0} \mid i l m \sigma\right)$ defined as multipole moments of the velocity of sphere $i$ relative to the incident flow taken at the center of sphere $i$. From this relation the matrix of multipole elements of the operator $\mathbf{Z}_{0}$ has been determined analytically. Due to the spherical symmetry of a single-sphere system, this matrix is diagonal in $l$ and $m$, and "nearly" diagonal in $\sigma$, with the only nondiagonal coupling between $\sigma=0$ and $\sigma=2$. As a result, it is easy to invert the matrix of the multipole elements of $\mathbf{Z}_{0}$, getting exact results also for the multipole elements of $\mathbf{Z}_{0}^{-1}$.

After discussing the problem of a single sphere, let us shift to a general case of $N$ spheres with given translational and rotational velocities $\mathbf{U}$ and $\boldsymbol{\Omega}$, immersed in an incident flow $\mathbf{v}_{0}(\mathbf{r})$. We are going to interpret Eq. (7) in terms of a scattering series (speaking differently, we will apply the method of reflections to the resistance problem ${ }^{1}$ ). To the lowest (zero) order in the scattering expansion the spheres are treated as independent from each other and the force density on the surface $S_{j}$ of each particle $j$ is calculated from Eq. (8) as $\mathbf{Z}_{0}(j)\left(\mathbf{w}_{j}-\mathbf{v}_{0}\right)$. In the first-order approximation, the Green operator $\mathbf{G}(i j)$ is applied, according to Eq. (4), to calculate the resulting flow contributions "going out" from the spheres $j \neq i$ and "coming in" onto the surface $S_{i}$. The sum of these contributions, $\Sigma_{j \neq i} \mathbf{G}(i j) \mathbf{Z}_{0}(j)\left(\mathbf{w}_{j}-\mathbf{v}_{0}\right)$, is the first-order correction to the ambient fluid flow which is coming in onto the surface of sphere $i$. With Eq. (8), the corresponding first-order additive correction to $\mathbf{f}_{i}$ is thus given as $\left[-\mathbf{Z}_{0}(i) \sum_{j \neq i} \mathbf{G}(i j) \mathbf{Z}_{0}(j)\left(\mathbf{w}_{j}-\mathbf{v}_{0}\right)\right]$. By iterating this procedure, the force density on the surface of each particle $i$ arises from the single-sphere operator $\left[-\mathbf{Z}_{0}(i)\right]$, applied to the sum of subsequent ambient flow field contributions. These flow contributions result from multiple scatterings. In a single scattering, the flow "produced" by a particle $j$ is "reflected" 1 or "scattered" 56 by the operator $\left[-\mathbf{G}(k j) \mathbf{Z}_{0}(j)\right]$ on the surface of particle $k$. In our notation, the multiple scattering series for $\mathbf{f}$ reads

$$
\mathbf{f}=\mathbf{Z}_{0}\left[1-\mathbf{G Z}_{0}+\mathbf{G Z}_{0} \mathbf{G} \mathbf{Z}_{0}-\ldots\right]\left(\mathbf{w}-\mathbf{v}_{0}\right) .
$$

The sum of this scattering series may be written as $\mathbf{f}=\mathbf{Z}_{0}\left(1+\mathbf{G Z}_{0}\right)^{-1}\left(\mathbf{w}-\mathbf{v}_{0}\right)$, in agreement with Eq. (7).

The conclusion from the multiple scattering expansion described above is the following. Even if we focus on the mobility problem for many spheres in quiescent fluid, we need the solution for the problem of a single particle in an arbitrary external ambient fluid flow.

Let us now describe how to calculate $\mathbf{f}$ explicitly. We start from the integral equation (6). By projection onto subsequent mutipole functions $\mid i \operatorname{lm} \sigma)$, Eq. (6) is reduced to an infinite system of linear algebraic equations, which relate the velocity multipoles to the force multipoles through the sum of two matrices. We have already outlined how to calculate the first matrix related to $\mathbf{Z}_{0}^{-1}$. The second one contains the irreducible multipole elements of the Green operator G, which depend on the dimensional distances between centers of particles, $r=\left|\mathbf{r}_{i}-\mathbf{r}_{j}\right|, i \neq j$, as

$$
\left(i l^{\prime} m^{\prime} \sigma^{\prime}|\mathbf{G}(i j)| j l m \sigma\right)=\frac{w\left(l^{\prime} m^{\prime} \sigma^{\prime}, l m \sigma\right)}{\eta r^{l+l^{\prime}+\sigma+\sigma^{\prime}-1}} .
$$

The coefficients $w\left(l^{\prime} m^{\prime} \sigma^{\prime}, l m \sigma\right)$ have been already calculated analytically in Ref. 62 . The hydrodynamic interactions among $N$ spheres are evaluated when the infinite set of multipole linear equations is truncated appropriately and once the corresponding finite-dimensional matrix is inverted. The resulting grand resistance matrix, which consists of multipole elements of the operator $\left(\mathbf{Z}_{0}^{-1}+\mathbf{G}\right)^{-1}$, determines the forces $\mathcal{F} \equiv\left(\mathcal{F}_{1}, \ldots, \mathcal{F}_{N}\right)$, torques $\mathcal{T} \equiv\left(\mathcal{T}_{1}, \ldots, \mathcal{T}_{N}\right)$, and higher force multipoles, exerted by the particles on the fluid, as linear combinations of velocity multipoles. Explicitly,

$$
\left(\begin{array}{c}
\mathcal{F} \\
\mathcal{T} \\
:
\end{array}\right)=\left(\begin{array}{ccc}
\boldsymbol{\zeta}^{t} & \boldsymbol{\zeta}^{t r} & . \\
\boldsymbol{\zeta}^{r t} & \boldsymbol{\zeta}^{r r} & \ldots \\
: & : & :
\end{array}\right)\left(\begin{array}{c}
\mathbf{U}-\mathbf{v}_{0} \\
\mathbf{\Omega}-\boldsymbol{\omega}_{0} \\
:
\end{array}\right),
$$

where $\quad \mathbf{v}_{0}=\left(\mathbf{v}_{01}, \ldots, \mathbf{v}_{0 N}\right), \quad \boldsymbol{\omega}_{0}=\left(\boldsymbol{\omega}_{01}, \ldots, \boldsymbol{\omega}_{0 N}\right), \quad \mathbf{v}_{0 i}$ $\equiv \mathbf{v}_{0}\left(\mathbf{r}_{i}\right)$, and $\boldsymbol{\omega}_{0 i} \equiv \frac{1}{2} \boldsymbol{\nabla} \times\left.\mathbf{v}_{0}(\mathbf{r})\right|_{\mathbf{r}=\mathbf{r}_{i}}$. The $\zeta^{p q}$ with $p, q=t$ or $r$, are Cartesian $3 N \times 3 N$ tensors corresponding to the translational and rotational components of the grand resistance tensor.

If there is no incident fluid flow, then the hydrodynamic interactions are described by a smaller $6 N \times 6 N$ friction tensor $\zeta$, given as 


$$
\boldsymbol{\zeta}=\left(\begin{array}{ll}
\boldsymbol{\zeta}^{t t} & \boldsymbol{\zeta}^{t r} \\
\boldsymbol{\zeta}^{r t} & \boldsymbol{\zeta}^{r r}
\end{array}\right)=\mathcal{P}\left[\mathbf{Z}_{0}^{-1}+\mathbf{G}\right]^{-1} \mathcal{P},
$$

where $\mathcal{P}$ denotes the projection operator on the multipole subspace determined by the Stokes monopoles and the antisymmetric dipoles (rotlets). In other words, the operator $\mathcal{P}$ projects onto the subspace spanned by the irreducible multipole functions with $l=1$ and $\sigma=0,1$.

The mobility tensor $\boldsymbol{\mu}$ is the inverse of the friction tensor, i.e.,

$$
\boldsymbol{\mu}=\left(\begin{array}{ll}
\boldsymbol{\mu}^{t t} & \boldsymbol{\mu}^{t r} \\
\boldsymbol{\mu}^{r t} & \boldsymbol{\mu}^{r r}
\end{array}\right)=\zeta^{-1}
$$

Instead of inverting $\zeta$ one can construct the mobility tensor directly as a scattering series ${ }^{63}$ equivalent to the sum of reflections fitted to the mobility problem, ${ }^{1}$

$$
\boldsymbol{\mu}=\boldsymbol{\mu}_{0}+\boldsymbol{\mu}_{0} \mathbf{Z}_{0} \frac{1}{1+\mathbf{G} \hat{\mathbf{Z}}_{0}} \mathbf{G Z}_{0} \boldsymbol{\mu}_{0}
$$

where

$$
\hat{\mathbf{Z}}_{0}=\mathbf{Z}_{0}-\mathbf{Z}_{0} \boldsymbol{\mu}_{0} \mathbf{Z}_{0} .
$$

Equation (14) can be derived as an algebraic identity from Eqs. (12) and (13). Let us explain the physical meaning of this formula. The scattering expansion for the mobility problem is similar to the one described before for the friction problem. The essential difference is that in the zero order of the expansion for the friction problem, the particle velocities are already reproduced exactly, and they are not corrected in the next steps. However, in the zero order of the expansion for the mobility problem, the forces and torques are already generated exactly. In subsequent reflections, the velocity fields are adjusted in such a way that in each subsequent order of the expansion the forces and torques are not changed. Therefore in the multiple scattering ${ }^{56,64}$ there appears the single-sphere resistance operator $\hat{\mathbf{Z}}_{0}$ rather than $\mathbf{Z}_{0}$. Indeed, according to definition (15), the operator $\hat{\mathbf{Z}}_{0}(i)$, applied to an arbitrary ambient flow, produces on the surface of particle $i$ a force density with zero force and zero torque.

To zero order in the scattering expansion, the spheres are treated as independent and $\boldsymbol{\mu}$ reduces to $\boldsymbol{\mu}_{0}$, the singlesphere mobility,

$$
\boldsymbol{\mu}_{0}=\left[\mathcal{P} \mathbf{Z}_{0} \mathcal{P}\right]^{-1} .
$$

In next order steps, the operator $\mathbf{Z}_{0} \boldsymbol{\mu}_{0}$ is applied to the forces and torques exerted on each sphere to get the force density on its surface, which in turn produces ambient flows on other particles with the use of the Green operator G. Next, these flows undergo multiple scattering with the operator [-G $\left.\hat{\mathbf{Z}}_{0}\right]$. Finally, the operator $\boldsymbol{\mu}_{0} \mathbf{Z}_{0}$ extracts from the ambient flows their lowest order multipoles, which are just the desired corrections to the particle velocities. Explicitly,

$$
\boldsymbol{\mu}=\boldsymbol{\mu}_{0}+\boldsymbol{\mu}_{0} \mathbf{Z}_{0}\left[1-\mathbf{G} \hat{\mathbf{z}}_{0}+\ldots\right] \mathbf{G} \mathbf{Z}_{0} \boldsymbol{\mu}_{0} .
$$

Note that the scattering expansion (17) of the mobility converges much faster ${ }^{63}$ than the corresponding expansion of the friction (9). Therefore, it is easier to determine the long- distance asymptotics of the mobility directly from its scattering expansion Eq. (17) rather than by inverting the friction tensor according to Eqs. (9) and (13).

\section{QUASI-TWO-DIMENSIONAL HYDRODYNAMIC INTERACTIONS BETWEEN $\boldsymbol{N}$ SPHERES}

Having recalled foundations of the low-Reynoldsnumber hydrodynamic interactions between many spheres in unbounded fluid, now we focus on a more complicated system of $N$ spheres immersed in a semi-infinite fluid bounded by a planar free interface. We adopt the system of coordinates with $z=0$ at the free interface and we assume that the fluid occupies the region $z>0$. The results for unbounded fluid, outlined in the preceding section, are now needed to apply the method of images. ${ }^{65}$

Within this method, the fluid is mentally extended to fill the whole space. The incident velocity field $\mathbf{v}_{0}$, given for $z$ $>0$, is now reflected with respect to the horizontal plane $z$ $=0$ into the lower half-space with $z<0$. In addition, the number of the particles is doubled: each real sphere $i$ from the upper half space is accompanied by an image sphere $i^{\prime}$ in the lower half space, with the position and force density vectors constructed by reflection in the plane $z=0$. Due to the inherent symmetry of the extended system, the freesurface boundary conditions (i.e., zero normal fluid velocity and zero tangential-normal components of the stress tensor) at $z=0$ are fulfilled automatically. Therefore for $z \geqslant 0$, the unbounded fictitious $2 \mathrm{~N}$-sphere system is described by the same hydrodynamics as the real system of $N$ spheres in the half space as illustrated in Fig. 2.

As it has been shown in Ref. 65, hydrodynamic interactions between $N$ real spheres in a fluid bounded by a flat interface are described by Eqs. (1)-(4), but with the integral kernel $\mathbf{T}$ identified now with a tensor $\mathbf{T}_{F}$, given as

$$
\mathbf{T}_{F}(\mathbf{r}, \overline{\mathbf{r}})=\mathbf{T}_{0}(\mathbf{r}-\overline{\mathbf{r}})+\mathbf{T}_{0}\left(\mathbf{r}-\overline{\mathbf{r}}^{\prime}\right) \cdot \mathcal{R}_{F},
$$

where $\boldsymbol{\mathcal { R }}_{F}=\mathbf{I}-2 \hat{\mathbf{z}} \hat{\mathbf{z}}$ is the operator of reflection with respect to the plane $z=0$, and $\hat{\mathbf{z}}$ is the unit vector normal to the boundary and pointing into the fluid. In particular, the reflection of a position vector $\overline{\mathbf{r}}=(\bar{x}, \bar{y}, \bar{z})$ is given by $\overline{\mathbf{r}}^{\prime}=\boldsymbol{\mathcal { R }}_{F} \overline{\mathbf{r}}$ $=(\bar{x}, \bar{y},-\bar{z})$. The subscript $F$ indicates a system bounded by a planar free interface. Definition (18) of the integral kernel $\mathbf{T}_{F}$ specifies $\mathbf{G}$, the Green operator in Eq. (6), which is therefore identified with the following $\mathbf{G}_{F}$,

$$
\mathbf{G}_{F}(i j)=\mathbf{G}_{0}\left(i i^{\prime}\right) \boldsymbol{\mathcal { R }}_{F} \delta_{i j}+\widetilde{\mathbf{G}}(i j)\left(1-\delta_{i j}\right),
$$
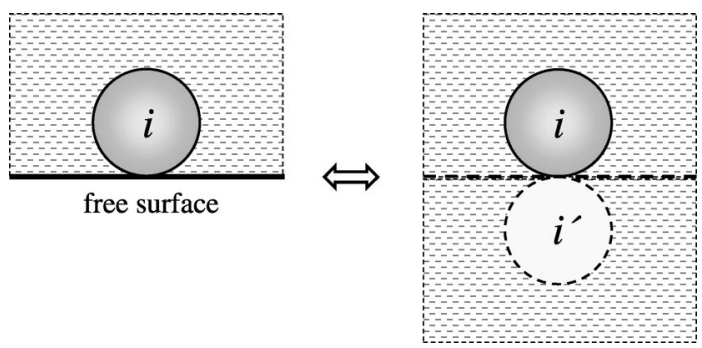

FIG. 2. The method of images for a sphere $i$, which touches the planar free surface. 
where

$$
\widetilde{\mathbf{G}}(i j)=\mathbf{G}_{0}(i j)+\mathbf{G}_{0}\left(i j^{\prime}\right) \boldsymbol{\mathcal { R }}_{F} .
$$

The inter-particle operator $\mathbf{G}_{F}(i j)$ consists of two terms. The first part describes the hydrodynamic interaction between a real sphere $i$ and its image $i^{\prime}$. The second part accounts for the interaction between a real sphere $i$ and a sphere doublet, i.e., another real sphere $j \neq i$ and its image $j^{\prime}$. For clarity, from now on we shall use $\mathbf{G}_{0}$ to denote the Green operator for an unbounded fluid with the Oseen kernel $\mathbf{T}_{0}$.

Hydrodynamic interactions between many spheres in a fluid bounded by a planar free surface have been analyzed in Ref. 50. In that work, the multipole expansion has been carried out with respect to the same multipole functions, which were previously used for the unbounded fluid. Therefore the multipole matrix elements of the single-sphere operator $\mathbf{Z}_{0}$ are the same as in the unbounded case, and the method to evaluate the multipole elements of the modified Green operator $\mathbf{G}_{F}$ and to perform the scattering expansion is a straightforward generalization of the one described in the preceding section. Thus the friction operator is given by the following analog of Eq. (12),

$$
\boldsymbol{\zeta}=\mathcal{P}\left[\mathbf{Z}_{0}^{-1}+\mathbf{G}_{F}\right]^{-1} \mathcal{P} .
$$

To interpret Eq. (21) as a multiple scattering series, we need to construct a single-sphere resistance operator other than $\mathbf{Z}_{0}$ from Eq. (8). This new operator, if applied to a given incident fluid flow around a real sphere $i$ moving with a given velocity, has to induce force densities not only on sphere $i$, but also on its image $i^{\prime}$. Therefore, by separating out from $\mathbf{G}_{F}$ the propagator between $i$ and $i^{\prime}$ according to Eq. (19), and combing it with $\mathbf{Z}_{0}(i)^{-1}$, we obtain the following one-sphere resistance operator in a fluid bounded by an interface,

$$
\widetilde{\mathbf{Z}}_{0}(i)=\left[\mathbf{Z}_{0}^{-1}(i)+\mathbf{G}_{0}\left(i i^{\prime}\right) \boldsymbol{R}_{F}\right]^{-1} .
$$

This expression may be interpreted as the two-particle hydrodynamic interactions between sphere $i$ and its image $i^{\prime}$ (c.f. Appendix A for the details). Indeed, $\widetilde{\mathbf{Z}}_{0}(i)$ is constructed from the grand resistance operator $\left[\mathbf{Z}_{0}^{-1}+\mathbf{G}_{0}\right]^{-1}$ for two spheres $i$ and $i^{\prime}$ as the sum of its components $i i$ and $i i^{\prime}$, the latter superposed with the reflection operator $\boldsymbol{\mathcal { R }}_{F}$. Therefore it is easy to evaluate the multipole elements of $\widetilde{\mathbf{Z}}_{0}(i)$ from the known multipole elements of the two-particle grand resistance operator, ${ }^{66}$ taking into account that the reflection operator $\boldsymbol{\mathcal { R }}_{F}$ acts on the multipole functions as

$$
\left.\left.\boldsymbol{\mathcal { R }}_{F} \mid i l m \sigma\right)=(-1)^{l+m+\sigma} \mid i^{\prime} l m \sigma\right) \text {. }
$$

So far, we have put no constraints on location of the spheres inside the half-infinite fluid. From now on we consider a genuine quasi-two-dimensional system in which the surfaces of all $N$ spheres touch the flat free interface at $z=0$, so that during the motion the sphere centers stay within the plane $z=a$. Unfortunately, there appears a difficulty with the construction of $\widetilde{\mathbf{Z}}_{0}(i)$ as described above if a sphere $i$ touches the interface. That is, due to lubrication effects, ${ }^{57}$ some of the multipole matrix elements of the operator $\widetilde{\mathbf{Z}}_{0}(i)$ turn out to be infinite in this limiting case. Of the particular

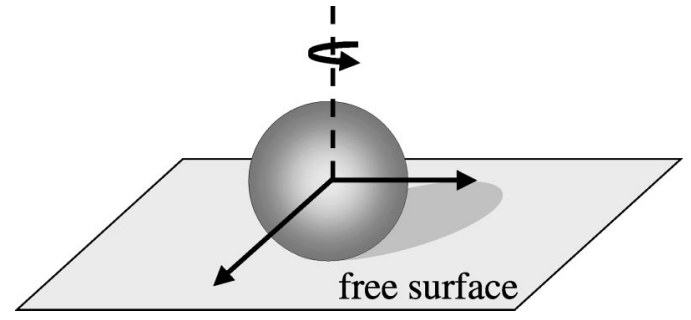

FIG. 3. Three degrees of freedom of a sphere, which touches the planar free surface.

interest are the multipole elements $\left(1 m \sigma\left|\widetilde{\mathbf{Z}}_{0}(i)\right| 1 m \sigma^{\prime}\right)$ with $m=0, \pm 1$ and $\sigma, \sigma^{\prime}=0,1$, which are needed to evaluate $\mathcal{P} \widetilde{\mathbf{Z}}_{0}(i) \mathcal{P}$. In Appendix A, the Cartesian form of $\mathcal{P} \widetilde{\mathbf{Z}}_{0}(i) \mathcal{P}$ is given in terms of the corresponding two-particle friction tensors, and it is shown there that the only divergent components are those related to the multipole elements $\left(100\left|\widetilde{\mathbf{Z}}_{0}(i)\right| 100\right)$ and $\left(1 \pm 11\left|\widetilde{\mathbf{Z}}_{0}(i)\right| 1 \pm 11\right)$. The divergence of these elements implies that if a real sphere touches its image, the lubrication effects do not allow either for its translation perpendicular to the interface,

$$
U_{i z}=0,
$$

or for its rotation along an axis parallel to the free surface,

$$
\Omega_{i x}=\Omega_{i y}=0 .
$$

Therefore, the motion of a real sphere touching a planar free surface is limited to translation parallel to the interface, and to rotation along the axis perpendicular to the interface. Only three degrees of freedom are left, as illustrated in Fig. 3.

For a quiescent fluid, i.e., when $\mathbf{v}_{0}(\mathbf{r})=0$, the translational and the rotational velocities of $N$ spheres, $\overline{\mathbf{U}} \equiv\left(U_{1 x}, U_{1 y}, \Omega_{1 z}, \ldots, U_{N x}, U_{N y}, \Omega_{N z}\right)$, can therefore be expressed in terms of the horizontal components of the external forces and the vertical components of the external torques, $\overline{\mathcal{F}} \equiv\left(\mathcal{F}_{1 x}, \mathcal{F}_{1 y}, \mathcal{T}_{1 z}, \ldots, \mathcal{F}_{N x}, \mathcal{F}_{N y}, \mathcal{T}_{N z}\right)$, by the linear relation,

$$
\overline{\mathbf{U}}=\bar{\mu} \overline{\mathcal{F}} .
$$

Our goal is to evaluate the quasi-two-dimensional mobility $\bar{\mu}$, which is a $3 N \times 3 N$ tensor and depends on relative positions of the sphere centers within the plane $z=a$. To account for the velocity constraints (24) and (25), we introduce an operator $\mathcal{P}_{F}$, which projects onto the allowed degrees of freedom. In the spherical multipole representation, $\mathcal{P}_{F}$ selects the multipoles with $(\operatorname{lm} \sigma) \in\{(110),(1-10),(101)\}$. These multipoles are symmetric under reflection with respect to the planar interface.

So as for the quasi-two-dimensional system, Eq. (21) is replaced by

$$
\overline{\boldsymbol{\zeta}}=\mathcal{P}_{F}\left[\widetilde{\mathbf{Z}}_{0}^{-1}+\widetilde{\mathbf{G}}\right]^{-1} \mathcal{P}_{F} .
$$

In analogy to Eq. (13), the quasi-two-dimensional mobility is given as

$$
\overline{\boldsymbol{\mu}}=\bar{\zeta}^{-1}
$$


The rhs of Eq. (27) is still only a formal expression and it remains to be shown that it is finite in the limit of the sphere surfaces approaching the interface. The problem is that some of the multipole elements of $\widetilde{\mathbf{Z}}_{0}(i)$ are divergent even for $l \sigma$ different from 10 or 11 . To perform the multipole scattering expansion, we need to cope with the divergence of these higher order multipole elements of $\widetilde{\mathbf{Z}}_{0}(i)$. That is, we need to construct the correct, finite resistance operator for a single sphere which touches the flat free surface.

The reason for the divergence is that the spherical multipole functions, which have been used do not match the symmetry of the system. In particular, these functions do not obey the boundary conditions imposed on the fluid flow at the planar interface. Therefore the idea developed in Ref. 67 is to construct a new set of multipole base functions, symmetric under reflection with respect to the interface. In this paper, we will simply use the "physical" sum of two spherical multipoles, $\mid i l m \sigma)$, centered at the particle $i$, and $\left.\mid i^{\prime} \operatorname{lm} \sigma\right)$, its reflected "twin," centered at the image $i^{\prime}$.

The sum automatically satisfies the boundary conditions at the free surface. With an appropriate normalization, the sum can be interpreted as the result of a projection operator $\mathbf{P}(i)$ applied to the spherical multipole function $\mid i \operatorname{lm} \sigma)$,

$$
\left.\left.\mathbf{P}(i) \mid i \operatorname{lm} \sigma)=\frac{1}{2}[\mid i \operatorname{lm} \sigma)+(-1)^{l+m+\sigma} \mid i^{\prime} \operatorname{lm} \sigma\right)\right] .
$$

The operator $\mathbf{P}(i)$ projects onto the subspace of functions symmetric under reflection with respect to the plane $z=0$. Its multipole matrix elements are determined from displacement theorems $^{62}$ (see Appendix B for the explicit formulas).

Let us consider again the general problem of $N$ spheres touching the free surface. Using the displacement theorems, ${ }^{62}$ one can show (see Ref. 67 for the details) that the Green operator $\widetilde{\mathbf{G}}$ in Eq. (20) has the form

$$
\widetilde{\mathbf{G}}=2 \mathbf{P}^{T} \mathbf{G}_{0} \mathbf{P},
$$

where the superscript $T$ denotes the transposition. Substituting this relation into Eq. (27) one gets

$$
\overline{\boldsymbol{\zeta}}=\mathcal{P}_{F}\left[\mathbf{Z}_{F}^{-1}+2 \mathbf{G}_{0}\right]^{-1} \mathcal{P}_{F},
$$

with a new one-sphere resistance operator

$$
\mathbf{Z}_{F}(i)=\mathbf{P}(i) \widetilde{\mathbf{Z}}_{0}(i) \mathbf{P}^{T}(i) .
$$

Using the "physical sums" of the multipole functions, we end up with $\mathbf{Z}_{F}$ and $\overline{\boldsymbol{\zeta}}$, which are finite even if the spheres touch the interface. Equation (31) has been obtained from Eq. (27) by applying in the scattering expansion of the operator $\bar{\zeta}$ the identity

$$
\mathcal{P}_{F}=\mathcal{P}_{F} \mathbf{P}=\mathbf{P}^{T} \mathcal{P}_{F} .
$$

Indeed, after an algebra we get

$$
\overline{\boldsymbol{\zeta}}=\mathcal{P}_{F}\left(\mathbf{P} \widetilde{\mathbf{Z}}_{0} \mathbf{P}^{T}-\mathbf{P} \widetilde{\mathbf{Z}}_{0} \mathbf{P}^{T} 2 \mathbf{G}_{0} \mathbf{P} \widetilde{\mathbf{Z}}_{0} \mathbf{P}^{T}+\ldots\right) \mathcal{P}_{F},
$$

which is just the scattering expansion of Eq. (31).

Now we are ready to construct the quasi-twodimensional mobility $\overline{\boldsymbol{\mu}}$, defined in Eq. (28), as a multipole scattering series, in a similar way as it has been done for an unbounded fluid. ${ }^{3,56}$ The quasi-two-dimensional system is described by the following analog of the algebraic identity (14) derived in Ref. 3 for a bulk suspension,

$$
\overline{\boldsymbol{\mu}}=\boldsymbol{\mu}_{F}+2 \boldsymbol{\mu}_{F} \mathbf{Z}_{F} \frac{1}{1+2 \mathbf{G}_{0} \hat{\mathbf{Z}}_{F}} \mathbf{G}_{0} \mathbf{Z}_{F} \boldsymbol{\mu}_{F}
$$

where the quasi-two-dimensional operator $\hat{\mathbf{Z}}_{F}$ is defined as

$$
\hat{\mathbf{Z}}_{F}=\mathbf{Z}_{F}-\mathbf{Z}_{F} \boldsymbol{\mu}_{F} \mathbf{Z}_{F},
$$

and $\boldsymbol{\mu}_{F}$ is the quasi-two-dimensional mobility operator of a single sphere touching a flat free surface,

$$
\boldsymbol{\mu}_{F}=\left[\mathcal{P}_{F} \mathbf{Z}_{F} \mathcal{P}_{F}\right]^{-1} \text {. }
$$

\section{QUASI-TWO-DIMENSIONAL MOBILITY OF A SINGLE SPHERE}

First, we evaluate the multipole matrix elements of the operators $\mathbf{Z}_{F}$ and $\boldsymbol{\mu}_{F}$ (the resulting values are given in Eqs. (B8)-(B20) of Appendix B). Then we transform from the spherical to the Cartesian form according to relation (B30) of Appendix B. Finally, we obtain the Cartesian quasi-twodimensional one-sphere mobility tensor,

$$
\boldsymbol{\mu}_{F}=\left(\begin{array}{ccc}
\mu_{F}^{t t} & 0 & 0 \\
0 & \mu_{F}^{t t} & 0 \\
0 & 0 & \mu_{F}^{r r}
\end{array}\right)
$$

with

$$
\begin{aligned}
& \mu_{F}^{t t} / \mu_{0}^{t t}=1.3799554, \\
& \mu_{F}^{r r} / \mu_{0}^{r r}=1.10920983 .
\end{aligned}
$$

Here $\mu_{0}^{t t}=[6 \pi \eta a]^{-1}$ and $\mu_{0}^{r r}=\left[8 \pi \eta a^{3}\right]^{-1}$ are the translational and rotational mobilities, respectively, of a sphere in an unbounded fluid. These numerical values have been calculated with the use of truncated multipole expansion up to the multipole order $L=80$ (see, e.g., Ref. 3 for the precise definition of $L$ ). The numerical accuracy is estimated from the requirement that the displayed digits remain unchanged when going from $L=20$ to $L=80$.

The translational and the rotational quasi-twodimensional one-sphere mobilities in Eqs. (39) and (40) are equal to the inverse of the drag and turn coefficients for two touching spheres, i.e., $\mu_{F}^{t t} / \mu_{0}^{t t}=\left[Y_{11}^{A}+Y_{12}^{A}\right]^{-1}$ and $\mu_{F}^{r r} / \mu_{0}^{r r}$ $=\left[X_{11}^{C}+X_{12}^{C}\right]^{-1}$ (here we follow the notation of Refs. 1, 57). Indeed, the values in Eqs. (39) and (40) agree with previous numerical results from Refs. 57 and 3 (i.e., $\mu_{F}^{t t} / \mu_{0}^{t t} \approx 1.3801$ and $\mu_{F}^{t t} / \mu_{0}^{t t} \approx 1.37996$, respectively). The comparison of the numerical value in Eq. (40) with the exact result given in Ref. 57, $\mu_{F}^{r r} / \mu_{0}^{r r}=4 /[3 \zeta(3)] \approx 1.1092098301$ (where $\zeta$ is the Riemann zeta function), provides us with a good test for the good numerical accuracy of our calculation. 


\section{LONG-DISTANCE CONTRIBUTION TO THE QUASI-TWO-DIMENSIONAL MOBILITY TENSOR FOR TWO SPHERES}

In this section, the general scheme developed in Sec. III is applied to determine the quasi-two-dimensional mobility of two distant spheres. For two spheres, the solution to Eq. (26) is given by

$$
\left(\begin{array}{c}
U_{1 x} \\
U_{1 y} \\
\Omega_{1 z}
\end{array}\right)=\overline{\boldsymbol{\mu}}_{11}\left(\begin{array}{c}
\mathcal{F}_{1 x} \\
\mathcal{F}_{1 y} \\
\mathcal{T}_{1 z}
\end{array}\right)+\overline{\boldsymbol{\mu}}_{12}\left(\begin{array}{c}
\mathcal{F}_{2 x} \\
\mathcal{F}_{2 y} \\
\mathcal{T}_{2 z}
\end{array}\right) .
$$

We assume that the distance between centers of both spheres, $r=\left|\mathbf{r}_{1}-\mathbf{r}_{2}\right|$, is significantly larger than their diameter $2 a$. Our goal is to calculate those leading long-distance terms in the expansion of $\overline{\boldsymbol{\mu}}_{11}$ and $\overline{\boldsymbol{\mu}}_{12}$, which scale as $1 / r^{n}$, with $n \leqslant 3$. The corresponding asymptotic expressions for the mobilities will be denoted as $\overline{\boldsymbol{\mu}}_{11}^{a}$ and $\overline{\boldsymbol{\mu}}_{12}^{a}$. So we select from Eq. (14) those terms in the scattering expansion of the mobility which include not more than three propagators $\mathbf{G}_{0}$, i.e.,

$$
\begin{aligned}
\overline{\boldsymbol{\mu}}_{11}= & \boldsymbol{\mu}_{F}+4 \boldsymbol{\mu}_{F} \mathbf{Z}_{F} \mathbf{G}_{0}(12) \hat{\mathbf{Z}}_{F} \mathbf{G}_{0}(21) \mathbf{Z}_{F} \boldsymbol{\mu}_{F}+\cdots, \\
\overline{\boldsymbol{\mu}}_{12}= & 2 \boldsymbol{\mu}_{F} \mathbf{Z}_{F} \mathbf{G}_{0}(12) \mathbf{Z}_{F} \boldsymbol{\mu}_{F} \\
& +8 \boldsymbol{\mu}_{F} \mathbf{Z}_{F} \mathbf{G}_{0}(12) \hat{\mathbf{Z}}_{F} \mathbf{G}_{0}(21) \hat{\mathbf{Z}}_{F} \mathbf{G}_{0}(12) \mathbf{Z}_{F} \boldsymbol{\mu}_{F}+\cdots,
\end{aligned}
$$

and we analyze their scaling in $1 / r$. From Eq. (36) it follows that $\mathcal{P}_{F} \hat{\mathbf{Z}}_{F}=\hat{\mathbf{Z}}_{F} \mathcal{P}_{F}=0$. Taking into account also Eq. (B11), we calculate that $\left(\operatorname{lm} \sigma\left|\hat{\mathbf{Z}}_{F}\right| l^{\prime} m^{\prime} \sigma^{\prime}\right)=0$, if $l+\sigma=1$ or $l^{\prime}+\sigma^{\prime}=1$. Due to this property, all the terms in Eqs. (42) and (43) which include $\hat{\mathbf{Z}}_{F}$ may be neglected. These terms behave as $1 / r^{4}$ and $1 / r^{7}$, respectively, since, according to Eq. (10), the multipole matrix elements of $\mathbf{G}_{0}(12)$ scale as $1 / r^{l+l^{\prime}+\sigma+\sigma^{\prime}-1}$. Therefore, $\overline{\boldsymbol{\mu}}_{11}^{a}$ is identified as the single sphere contribution,

$$
\overline{\boldsymbol{\mu}}_{11}^{a}=\boldsymbol{\mu}_{F},
$$

with the Cartesian form of $\boldsymbol{\mu}_{F}$ given already in Eqs. (38)(40).

Let us derive next the asymptotic expression $\boldsymbol{\mu}_{12}^{a}$. Note that $\overline{\boldsymbol{\mu}}_{12}^{a}$ contains only the leading multipole elements of the first term in Eq. (43), i.e., of $2 \boldsymbol{\mu}_{F} \mathbf{Z}_{F} \mathbf{G}_{0}(12) \mathbf{Z}_{F} \boldsymbol{\mu}_{F}$. Their dependence on $r$ is determined by the multipole matrix elements of $\mathbf{G}_{0}$ according to Eq. (10). Therefore it is sufficient to calculate $w\left(l^{\prime} m^{\prime} \sigma^{\prime}, l m \sigma\right)$ only for the multipoles with $l^{\prime}+\sigma^{\prime}+l+\sigma \leqslant 4$ and $m, m^{\prime}= \pm 1$, and to calculate $\left(\operatorname{lm} \sigma \mid \mathbf{Z}_{F} 1 m 0=0\right.$ only for the multipoles which satisfy $l+\sigma \leqslant 3, m= \pm 1$.

To construct the Cartesian form of the operator $\overline{\boldsymbol{\mu}}_{12}^{a}$, we first calculate its multipole elements [for the details see Eqs. (B23)-(B28) in Appendix B]. Next, we transform these elements into Cartesian form using Eq. (B30) of Appendix B. We choose the $x$ axis in the same way as in Ref. 57-along the vector $\left(\mathbf{r}_{2}-\mathbf{r}_{1}\right)$, which joins the particle centers. Therefore we have to put $\phi=\pi$ in Eqs. (B23)-(B26). Finally, we get the asymptotic long-distance form of $\overline{\boldsymbol{\mu}}_{12}$ as

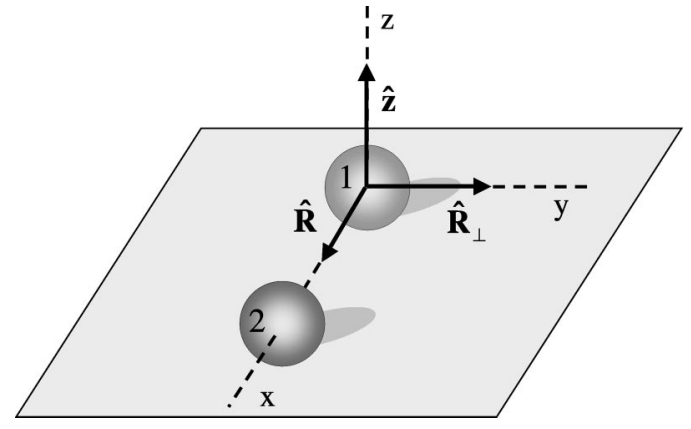

FIG. 4. The system of coordinates used in Eqs. (45)-(48).

$$
\begin{aligned}
& \overline{\boldsymbol{\mu}}_{12}^{a}=\mu_{0}^{t t}\left(\frac{1}{R}\left(\begin{array}{ccc}
\frac{3}{2} & 0 & 0 \\
0 & \frac{3}{4} & 0 \\
0 & 0 & 0
\end{array}\right)\right. \\
&-\frac{1}{R^{3}}\left(\begin{array}{ccc}
1.159862 \ldots & 0 & 0 \\
0 & 0.111686 \ldots & 0 \\
0 & 0 & 0
\end{array}\right) \\
&+ \frac{\mu_{0}^{r t}}{4 R^{2}}\left(\begin{array}{ccc}
0 & 0 & 0 \\
0 & 0 & -1 \\
0 & 1 & 0
\end{array}\right)-\frac{\mu_{0}^{r r}}{8 R^{3}}\left(\begin{array}{lll}
0 & 0 & 0 \\
0 & 0 & 0 \\
0 & 0 & 1
\end{array}\right),
\end{aligned}
$$

with the dimensionless interparticle distance $R=r /(2 a)$. The notion of $\mu_{0}^{t t}$ and $\mu_{0}^{r r}$ has been already explained in the preceding section; $\mu_{0}^{r t}=\left[4 \pi \eta a^{2}\right]^{-1}$ is the rotationaltranslational mobility normalization coefficient. Our final result (45) can be alternatively expressed in terms of $3 \times 3$ Cartesian two-sphere mobility tensors, which are defined and labeled in analogy to Eq. (13), namely the rotationalrotational mobility $\overline{\boldsymbol{\mu}}_{12}^{a, r r}$, the rotational-translational mobility $\overline{\boldsymbol{\mu}}_{12}^{a, r t}$, the translational-rotational mobility $\overline{\boldsymbol{\mu}}_{12}^{a, t r}$, and the translational-translational mobility $\overline{\boldsymbol{\mu}}_{12}^{a, t t}$. We get

$$
\begin{aligned}
\overline{\boldsymbol{\mu}}_{12}^{a, r r} / \mu_{0}^{r r}= & -\frac{1}{8 R^{3}} \hat{\mathbf{z}} \hat{\mathbf{z}}, \\
\overline{\boldsymbol{\mu}}_{12}^{a, r t} / \mu_{0}^{r t}= & \frac{1}{4 R^{2}} \hat{\mathbf{z}} \hat{\mathbf{R}}, \quad \overline{\boldsymbol{\mu}}_{12}^{a, t r} / \mu_{0}^{r t}=-\frac{1}{4 R^{2}} \hat{\mathbf{R}} \hat{\mathbf{z}}, \\
\overline{\boldsymbol{\mu}}_{12}^{a, t t} / \mu_{0}^{t t}= & \frac{3}{4 R}\left[2 \hat{\mathbf{R}} \hat{\mathbf{R}}+\hat{\mathbf{R}}_{\perp} \hat{\mathbf{R}}_{\perp}\right]-\frac{1}{R^{3}}[1.159862 \ldots \hat{\mathbf{R}} \hat{\mathbf{R}} \\
& \left.+0.111686 \ldots \hat{\mathbf{R}}_{\perp} \hat{\mathbf{R}}_{\perp}\right],
\end{aligned}
$$

with the unit vectors $\hat{\mathbf{z}}, \hat{\mathbf{R}}$, and $\hat{\mathbf{R}}_{\perp}$ depicted in Fig. 4 .

Note that $\hat{\mathbf{z}}$ is perpendicular to the planar interface spanned by $\hat{\mathbf{R}} \equiv\left(\mathbf{r}_{2}-\mathbf{r}_{1}\right) / r$ and $\hat{\mathbf{R}}_{\perp} \equiv \hat{\mathbf{z}} \times \hat{\mathbf{R}}$. Moreover, $\hat{\mathbf{z}}$ points into the fluid and $\hat{\mathbf{R}}_{\perp}$ is perpendicular to the line of sphere centers. 
TABLE I. The $1 / R^{3}$ term in the long-distance expansion of the translationaltranslational mobility $\boldsymbol{\mu}_{12}^{t t} / \mu_{0}^{t t}$ for two spheres. Comparison of the quasi-twodimensional motion along a planar free surface (this work) with the motion in a three-dimensional unbounded system (the Rotne-Prager expression, c.f. Refs. 1 and 68).

\begin{tabular}{lcc}
\hline \hline & $\begin{array}{c}\text { Quasi-two-dimensional } \\
\text { system }\end{array}$ & $\begin{array}{c}\text { Three-dimensional } \\
\text { system }\end{array}$ \\
\hline Longitudinal & $-\frac{1.159862 \ldots}{R^{3}}$ & $-\frac{0.125}{R^{3}}$ \\
Transverse & $-\frac{0.111686 \ldots}{R^{3}}$ & $\frac{0.0625}{R^{3}}$ \\
\hline \hline
\end{tabular}

\section{CONCLUSIONS}

The main result of this paper is calculation of the longdistance leading terms for the quasi-two-dimensional mobility of two spheres moving along a planar free surface. That is, the expansion of the mobility tensor in inverse powers of the interparticle distance $R$ has been carried out and all the coefficients next to $1 / R, 1 / R^{2}$, and $1 / R^{3}$ have been evaluated.

The outcome, given in Eqs. (45)-(48), should be now compared with the corresponding asymptotic form of the three-dimensional mobility of two spheres moving without constraints in an unbounded fluid. We have shown in Eqs. (46)-(48) that the lowest order terms in the expansion of the rotational-rotational $\left(\sim 1 / R^{3}\right)$, rotational-translational $\left(\sim 1 / R^{2}\right)$, and translational-translational $(\sim 1 / R)$ parts of the quasi-two-dimensional mobility $\boldsymbol{\mu}_{12}^{a}$ are twice as large as their three-dimensional counterparts (compare, e.g., with Ref. 1). This result may be easily understood qualitatively within the method of images. To the lowest order of the expansion, the position of sphere 2 as "seen" from the distant sphere 1, coincides with the position of its image $2^{\prime}$. Therefore the presence of the image effectively doubles the forces and torques acting on sphere 2 , and also the resulting translational and angular velocities of sphere 1 .

However, the higher order correction $\left(\sim 1 / R^{3}\right)$ to the translational-translational quasi-two-dimensional mobility $\boldsymbol{\mu}_{12}^{t t}$ in Eq. (48) cannot be interpreted in a simple way. In Table I, the $1 / R^{3}$ term in the expansion of $\boldsymbol{\mu}_{12}^{t t} / \mu_{0}^{t t}$ is compared with the corresponding term in the expansion of the mobility for three-dimensional unbounded system (c.f. the Rotne-Prager expression ${ }^{68}$ ). For the quasi-two-dimensional system, the longitudinal $1 / R^{3}$ term is of the same sign, but one order of magnitude larger than its bulk counterpart; the quasi-two-dimensional transverse term is of the opposite sign and about twice as large as its bulk counterpart.
The question arises what is the accuracy of the longdistance approximation developed in this work for a typical experimental system. As an example we consider the measurements from Ref. 31, with the sphere centers separated by a distance equal to three diameters, $R=3$. Using numerically exact results for the translational-translational mobility (c.f. Ref. 67), in Table II we estimate the accuracy of the $1 / R$ and $1 / R^{3}$ approximations.

In particular, from Table II it follows that for two spheres separated by $R \sim 3$, only three digits in the longitudinal mobility coefficient and four digits in the transverse one are meaningful in the approximate Eq. (45). In general, as illustrated by the example in Table II, for the longitudinal coefficient of $\boldsymbol{\mu}_{12}^{t t}$ the convergence of its $1 / R$ expansion is one order of magnitude slower than for the transverse one.

\section{ACKNOWLEDGMENTS}

G.N. is grateful to the Center of Excellence for Advanced Materials and Structures for generous financial support, and to the members of the Institute of Fundamental Technological Research (Polish Academy of Sciences) for their warm hospitality during his stay in Warsaw.

\section{APPENDIX A: THE CARTESIAN FORM OF THE OPERATOR $\mathcal{P} \tilde{Z}_{0}(i) \mathcal{P}$}

Let us interpret the one-sphere resistance operator $\widetilde{\mathbf{Z}}_{0}(i)$ in terms of the two-particle hydrodynamic interactions between sphere $i$ and its image $i^{\prime}$. To this goal, consider Eq. (7) for such a two-particle system. For $z<0$, the spurious incident fluid flow $\mathbf{v}_{0}^{\prime}$, the force density $\mathbf{f}_{i^{\prime}}$, and velocity field $\mathbf{w}_{i}$, of the image sphere are obtained by reflection with respect to the plane $z=0$ of the corresponding vectors of the real system. Denoting the reflection operator as $\mathcal{R}_{F}$, we write $\mathbf{v}_{0}^{\prime}=\boldsymbol{\mathcal { R }}_{F} \mathbf{v}_{0}, \mathbf{f}_{i^{\prime}}=\boldsymbol{\mathcal { R }}_{F} \mathbf{f}_{i}, \mathbf{w}_{i^{\prime}}=\boldsymbol{\mathcal { R }}_{F} \mathbf{w}_{i}$. Then Eq. (7) reads

$$
\left(\begin{array}{c}
\mathbf{f}_{i} \\
\boldsymbol{\mathcal { R }}_{F} \mathbf{f}_{i}
\end{array}\right)=\left(\begin{array}{cc}
{\left[\mathbf{Z}\left(i i^{\prime}\right)\right]_{i i}} & {\left[\mathbf{Z}\left(i i^{\prime}\right)\right]_{i i^{\prime}}} \\
{\left[\mathbf{Z}\left(i i^{\prime}\right)\right]_{i^{\prime} i}} & {\left[\mathbf{Z}\left(i i^{\prime}\right)\right]_{i^{\prime} i^{\prime}}}
\end{array}\right)\left(\begin{array}{c}
\left(\mathbf{w}_{i}-\mathbf{v}_{0}\right) \\
\boldsymbol{\mathcal { R }}_{F}\left(\mathbf{w}_{i}-\mathbf{v}_{0}\right)
\end{array}\right),
$$

where

$$
\mathbf{Z}=\left(\mathbf{Z}_{0}^{-1}+\mathbf{G}\right)^{-1}
$$

stands for the two-particle grand resistance operator. In Eq. (A1), this operator, denoted explicitly as $\mathbf{Z}\left(i i^{\prime}\right)$, has been decomposed into four parts, labeled by the subscripts $i$ and $i^{\prime}$, according to the notation introduced in Refs. 1 and 57. From Eq. (A1) it follows that

TABLE II. Translational-translational quasi-two-dimensional mobility $\boldsymbol{\mu}_{12}^{t t} / \mu_{0}^{t t}$ for two spheres with their centers separated by three diameters, $R=3$. Comparison of the two leading long-distance terms with the numeri-

\begin{tabular}{|c|c|c|c|c|c|c|}
\hline & \multirow[b]{2}{*}{ Total } & \multirow[b]{2}{*}{$1 / R$ term } & \multicolumn{2}{|c|}{$1 / R^{3}$ term } & \multicolumn{2}{|c|}{ The other terms } \\
\hline & & & Value & $\%$ of total & Value & $\%$ of total \\
\hline Longitudinal & 0.463 & 0.500 & -0.043 & $9.3 \%$ & 0.006 & $1.3 \%$ \\
\hline Transverse & 0.24632 & 0.25000 & -0.00414 & $1.7 \%$ & 0.00046 & $0.19 \%$ \\
\hline
\end{tabular}
cally exact result, obtained for the multipole order $L=40$ (c.f. Ref. 67). 


$$
\widetilde{\mathbf{Z}}_{0}(i)=\left[\mathbf{Z}\left(i i^{\prime}\right)\right]_{i i}+\left[\mathbf{Z}\left(i i^{\prime}\right)\right]_{i i}, \boldsymbol{R}_{F} .
$$

To apply directly well-known results for the hydrodynamic two-sphere problem in infinite fluid, ${ }^{1,57}$ we relabel sphere $i$ as 1 and its image $i^{\prime}$ as 2 . The Cartesian form of the operator $\mathcal{P} \widetilde{\mathbf{Z}}_{0}(1) \mathcal{P}$ is constructed from the friction tensors $\zeta_{11}$ $=\mathcal{P}[\mathbf{Z}(12)]_{11} \mathcal{P}$ and $\zeta_{12}=\mathcal{P}[\mathbf{Z}(12)]_{12} \mathcal{P}$ as follows:

$$
\mathcal{P} \widetilde{\mathbf{Z}}_{0}(1) \mathcal{P}=\left(\begin{array}{ll}
\boldsymbol{\zeta}_{11}^{t t}+\boldsymbol{\zeta}_{12}^{t t} \boldsymbol{\mathcal { R }}_{F} & \boldsymbol{\zeta}_{11}^{t r}+\boldsymbol{\zeta}_{12}^{t r} \boldsymbol{\mathcal { R }}_{F} \\
\boldsymbol{\zeta}_{11}^{r t}+\boldsymbol{\zeta}_{12}^{r t} \boldsymbol{\mathcal { R }}_{F} & \boldsymbol{\zeta}_{11}^{r r}+\boldsymbol{\zeta}_{12}^{r r} \boldsymbol{\mathcal { R }}_{F}
\end{array}\right) .
$$

Applying the operator $\boldsymbol{\mathcal { R }}_{F}$, specified explicitly in Eq. (23), we get

$$
\begin{aligned}
& \boldsymbol{\zeta}_{11}^{t t}+\boldsymbol{\zeta}_{12}^{t t} \boldsymbol{\mathcal { R }}_{F}=6 \pi \eta a\left(\begin{array}{ccc}
Y_{11}^{A}+Y_{12}^{A} & 0 & 0 \\
0 & Y_{11}^{A}+Y_{12}^{A} & 0 \\
0 & 0 & X_{11}^{A}-X_{12}^{A}
\end{array}\right), \\
& \boldsymbol{\zeta}_{11}^{t r}+\boldsymbol{\zeta}_{12}^{t r} \boldsymbol{R}_{F}=4 \pi \eta a^{2}\left(\begin{array}{ccc}
0 & -Y_{11}^{B}-Y_{12}^{B} & 0 \\
Y_{11}^{B}+Y_{12}^{B} & 0 & 0 \\
0 & 0 & 0
\end{array}\right) \\
&=\left[\boldsymbol{\zeta}_{11}^{r t}+\boldsymbol{\zeta}_{12}^{r t} \boldsymbol{\mathcal { R }}_{F}\right]^{T},
\end{aligned}
$$

$\boldsymbol{\zeta}_{11}^{r r}+\boldsymbol{\zeta}_{12}^{r r} \mathcal{R}_{F}$

$$
=8 \pi \eta a^{3}\left(\begin{array}{ccc}
Y_{11}^{C}-Y_{12}^{C} & 0 & 0 \\
0 & Y_{11}^{C}-Y_{12}^{C} & 0 \\
0 & 0 & X_{11}^{C}+X_{12}^{C}
\end{array}\right) .
$$

Here we follow the standard notation of Refs. 1 and 57, where $X_{1 i}^{A}, Y_{1 i}^{A}, Y_{1 i}^{B}, X_{1 i}^{C}, Y_{1 i}^{C}, i=1,2$, have been defined and calculated as functions of $\xi=\left|\mathbf{r}_{1}-\mathbf{r}_{2}\right| / a-2$, the separation between the surfaces of spheres 1 and 2 .

In the limit of touching spheres, i.e., for $\xi \rightarrow 0$, the combinations $Y_{11}^{A}+Y_{12}^{A}, Y_{11}^{B}+Y_{12}^{B}$, and $X_{11}^{C}+X_{12}^{C}$ are finite, but all the other combinations in Eqs. (A4)-(A7) diverge because of lubrication effects, ${ }^{1,57}$ according to

$$
\begin{aligned}
& X_{11}^{A}-X_{12}^{A}=\frac{1}{2 \xi}+\frac{9}{20} \ln \xi^{-1}+\mathcal{O}(1), \\
& Y_{11}^{C}-Y_{12}^{C}=\frac{3}{20} \ln \xi^{-1}+\mathcal{O}(1) .
\end{aligned}
$$

In terms of the spherical multipole moments, the above result means that the matrix elements $\left(100\left|\widetilde{\mathbf{Z}}_{0}(i)\right| 100\right)$ and $\left(1 \pm 11\left|\widetilde{\mathbf{Z}}_{0}(i)\right| 1 \pm 11\right)$ diverge.

From Eqs. (A8) and (A9) it follows that a sphere which touches a flat free surface neither translates perpendicularly to the interface nor rotates along any axis parallel to the interface, no matter how large are the external force and torque applied to it.

\section{APPENDIX B: MULTIPOLE MATRIX ELEMENTS OF THE OPERATORS $P, G_{0}, Z_{F}, \mu_{F}$, AND $\bar{\mu}_{12}$, USED TO OBTAIN THE CARTESIAN QUASI-TWO- DIMENSIONAL MOBILITY OF TWO SPHERES}

The multipole elements of the operator $\mathbf{P}$ have been evaluated with the use of the displacement theorems from Ref. 62 (the details are given in Ref. 67). They are diagonal in $m, m^{\prime}$ and may be written as

$$
\begin{aligned}
\left(l m \sigma|\mathbf{P}| l^{\prime} m^{\prime} \sigma^{\prime}\right) & \\
= & \frac{\delta_{m m^{\prime}}}{2}\left[\delta_{l l^{\prime}} \delta_{\sigma \sigma^{\prime}}+(-1)^{l+m+\sigma}\right. \\
& \left.\times \frac{n_{l^{\prime} m^{\prime}}}{n_{l m}} S^{++}\left(\mathbf{r}_{i i^{\prime}} ; l^{\prime} m \sigma^{\prime}, l m \sigma\right)\right],
\end{aligned}
$$

where $\mathbf{r}_{i i^{\prime}}=2 a \hat{\mathbf{z}}$ and

$$
n_{l m}=\frac{4 \pi}{2 l+1} \sqrt{\frac{(l+m) !}{(l-m) !}} .
$$

The multipole elements $S^{++}\left(\mathbf{r}_{i i^{\prime}} ; l^{\prime} m \sigma^{\prime}, \operatorname{lm} \sigma\right)$ are the same as those defined and calculated in Ref. 62, but with the following misprint corrected: for $\sigma=2$, the sum in Eq. (3.2a) of Ref. 62 should contain one more term with $l^{\prime}=l+1$ and $\sigma^{\prime}=0$, which is evaluated according to Eq. (3.7) in Ref. 62 .

The multipole elements of the Green operator $\mathbf{G}_{0}(12)$, defined in Eq. (10), have been calculated in Ref. 62 and expressed in terms of functions $S^{+-}\left(\mathbf{r} ; l^{\prime} m \sigma^{\prime}, l m \sigma\right)$, where $\mathbf{r}=\mathbf{r}_{12}$ joins the centers of particles 2 and 1 . Our coefficients $w\left(l^{\prime} m^{\prime} \sigma^{\prime}, l m \sigma\right)$ in Eq. (10) are related to $S^{+-}$by the formula,

$$
\frac{w\left(l^{\prime} m^{\prime} \sigma^{\prime}, l m \sigma\right)}{r^{l+l^{\prime}+\sigma+\sigma^{\prime}-1}}=\frac{n_{l^{\prime} m^{\prime}}}{n_{l m}} S^{+-}\left(\mathbf{r} ; l^{\prime} m \sigma^{\prime}, \operatorname{lm} \sigma\right)
$$

Using the normalization of Ref. 50, we can benefit from the following symmetry relations,

$$
\begin{aligned}
& w\left(l^{\prime} m^{\prime} \sigma^{\prime}, l m \sigma\right)=(-1)^{l+l^{\prime}+\sigma+\sigma^{\prime}} w^{*}\left(\operatorname{lm} \sigma, l^{\prime} m^{\prime} \sigma^{\prime}\right), \\
& w\left(l^{\prime}-m^{\prime} \sigma^{\prime}, l-m \sigma\right) \\
& \quad=(-1)^{m+m^{\prime}+\sigma+\sigma^{\prime}} w^{*}\left(l^{\prime} m^{\prime} \sigma^{\prime}, \operatorname{lm} \sigma\right),
\end{aligned}
$$

where the asterisk denotes complex conjugation. The coefficients $S^{+-}\left(\mathbf{r} ; l^{\prime} m \sigma^{\prime}, l m \sigma\right)$ depend on the spherical harmonics $Y_{L M}(\theta, \phi)$, with angles $\theta, \phi$ specifying the direction of the vector $\mathbf{r}$ with respect to the vertical axis. For the quasitwo-dimensional system considered in this work, the motion is always horizontal, i.e. $\mathbf{r} \perp \hat{\mathbf{z}}$, so that $\theta=\pi / 2$. In this case $w\left(l^{\prime} m^{\prime} \sigma^{\prime}, l m \sigma\right)=0$, if $l^{\prime}+m^{\prime}+\sigma^{\prime}+l+m+\sigma$ is odd.

Now let us briefly discuss the one-sphere resistance operator $\mathbf{Z}_{0}$. Due to the spherical symmetry, its multipole elements are diagonal in $l l^{\prime}$, diagonal in $\mathrm{mm}^{\prime}$, and they do not depend on $m$,

$\left(l m \sigma\left|\mathbf{Z}_{0}\right| l^{\prime} m^{\prime} \sigma^{\prime}\right)=\delta_{m m^{\prime}} \delta_{l l^{\prime}} \eta(2 a)^{2 l+\sigma+\sigma^{\prime}-1} z_{0}\left(l \sigma, l \sigma^{\prime}\right)$.

Moreover, $z_{0}\left(l \sigma, l \sigma^{\prime}\right)$ is symmetric with respect to interchange of $\sigma$ and $\sigma^{\prime}$, and it vanishes when $\sigma+\sigma^{\prime}$ is an odd 
integer. Its values have been given, e.g., in Refs. 50 and 59. In particular, $z_{0}(10,10)=9 / 4, z_{0}(11,11)=3 / 4$ and $z_{0}(10,12)$ $=45 / 16$.

The resistance operator $\mathbf{Z}_{F}$, defined by Eq. (32) for a single sphere moving along a planar free surface, includes the hydrodynamic interactions between the sphere and its image, as evaluated by the algorithm developed in Ref. 66. The multipole matrix elements of $\mathbf{Z}_{F}$ are diagonal in $\mathrm{mm}^{\prime}$, (like in case of $\mathbf{Z}_{0}$ ), but nondiagonal in $l l^{\prime}$, since there is no spherical symmetry in the quasi-two-dimensional case. Therefore,

$$
\begin{aligned}
& \left(\operatorname{lm} \sigma\left|\mathbf{Z}_{F}\right| l^{\prime} m^{\prime} \sigma^{\prime}\right) \\
& \quad=\delta_{m m^{\prime}} \eta(2 a)^{l+l^{\prime}+\sigma+\sigma^{\prime}-1} z_{F}\left(\operatorname{lm} \sigma, l^{\prime} m \sigma^{\prime}\right),
\end{aligned}
$$

and $z_{F}$ displays further the following symmetry properties:

$$
\begin{aligned}
& z_{F}\left(l^{\prime} m \sigma^{\prime}, l m \sigma\right)=z_{F}\left(\operatorname{lm} \sigma, l^{\prime} m \sigma^{\prime}\right) \\
& z_{F}\left(l-m \sigma, l^{\prime}-m \sigma^{\prime}\right)=(-1)^{\sigma+\sigma^{\prime}} z_{F}\left(\operatorname{lm} \sigma, l^{\prime} m \sigma^{\prime}\right), \\
& z_{F}(100, l m \sigma)=0 .
\end{aligned}
$$

The explicit numerical values of $z_{F}$, used in this paper, are

$$
\begin{aligned}
& z_{F}(101,101)=0.67615701, \\
& z_{F}(110,110)=1.6304875, \\
& z_{F}(110,111)=0.8152437, \\
& z_{F}(110,112)=7.679014, \\
& z_{F}(110,210)=-1.822940, \\
& z_{F}(110,211)=-2.06797116, \\
& z_{F}(110,310)=3.280610 .
\end{aligned}
$$

These numerical values have been calculated using truncated multipole expansion up to the multipole order $L=80$ (see, e.g., Ref. 3 for the precise definition of $L$ ). The numerical accuracy is estimated from the requirement that the displayed digits remain unchanged when going from $L=20$ to $L=80$.

With the above results, it is straightforward to apply Eq. (37), and to check that the only nonvanishing multipole elements of $\boldsymbol{\mu}_{F}$ are given by

$$
\begin{aligned}
\left(110\left|\boldsymbol{\mu}_{F}\right| 110\right) & =\left(1-10\left|\boldsymbol{\mu}_{F}\right| 1-10\right) \\
& =\frac{0.6133135}{2 a \eta} \\
& =1.3799554\left(110\left|\boldsymbol{\mu}_{0}\right| 110\right), \\
\left(101\left|\boldsymbol{\mu}_{F}\right| 101\right) & =\frac{1.47894644}{(2 a)^{3} \eta} \\
& =1.10920983\left(101\left|\boldsymbol{\mu}_{0}\right| 101\right) .
\end{aligned}
$$

To calculate the multipole elements, which form the asymptotic two-sphere mobility matrix, we have to analyze the scattering sequence $2 \boldsymbol{\mu}_{F} \mathbf{Z}_{F} \mathbf{G}_{0}(12) \mathbf{Z}_{F} \boldsymbol{\mu}_{F}$. We obtain a very simple expression for the rotational-rotational components, i.e. $\left(l^{\prime} m^{\prime} \sigma^{\prime}\right),(l m \sigma)=(101), \quad$ and for the translational-rotational components, i.e. $\left(l^{\prime} m^{\prime} \sigma^{\prime}\right)=(1 \pm 10)$ and $(\operatorname{lm} \sigma)=(101)$, that is

$$
\begin{aligned}
\left(1 m^{\prime} \sigma^{\prime}\left|\overline{\boldsymbol{\mu}}_{12}\right| 1 m \sigma\right)= & 2 \frac{w\left(1 m^{\prime} \sigma^{\prime}, 1 m \sigma\right)}{\eta r^{\sigma^{\prime}+\sigma+1}}, \\
& \text { for } m=0 \text { or } m^{\prime}=0 .
\end{aligned}
$$

The $1 / r$ contribution to the translational-translational mobility also has the form of Eq. (B21). To get the remaining long-distance part of the translational-translational mobility proportional to $1 / r^{3}$, we need to sum up several multipole elements of $\mathbf{G}_{0}$, which contribute to the scattering sequence $2 \boldsymbol{\mu}_{F} \mathbf{Z}_{F} \mathbf{G}_{0}(12) \mathbf{Z}_{F} \boldsymbol{\mu}_{F}$,

$$
\begin{aligned}
\left(1 m^{\prime} 0\left|\overline{\boldsymbol{\mu}}_{12}\right| 1 m 0\right)-2 \frac{w\left(1 m^{\prime} 0,1 m 0\right)}{\eta r}= & \frac{2}{\eta r^{3}} \sum_{(l \sigma) \in A} \frac{w\left(1 m^{\prime} 0, \operatorname{lm} \sigma\right) z_{F}(\operatorname{lm} \sigma, 1 m 0)+w\left(\operatorname{lm} m^{\prime} \sigma, 1 m 0\right) z_{F}\left(\operatorname{lm} m^{\prime} \sigma, 1 m^{\prime} 0\right)}{z_{F}(110,110)} \\
& +\frac{2}{\eta r^{3}} \sum_{\left(l^{\prime} \sigma^{\prime}\right),(l \sigma) \in B} \frac{w\left(l^{\prime} m^{\prime} \sigma^{\prime}, \operatorname{lm} \sigma\right) z_{F}\left(l^{\prime} m^{\prime} \sigma^{\prime}, 1 m^{\prime} 0\right) z_{F}(\operatorname{lm} \sigma, 1 m 0)}{z_{F}^{2}(110,110)} \\
& \text { for } m, m^{\prime}= \pm 1 .
\end{aligned}
$$

Here $A=\{(12),(21),(30)\}, B=\{(11),(20)\}$, and the matrix elements $z_{F}$ and $w$ are given in Eqs. (B9)-(B18) and Eqs. (B3)-(B6), respectively. After some algebra, we get

$$
\begin{aligned}
& \left(101\left|\overline{\boldsymbol{\mu}}_{12}\right| 101\right)=-\frac{1}{6 \eta r^{3}}, \\
& \left(101\left|\overline{\boldsymbol{\mu}}_{12}\right| 110\right)=\frac{\exp (i \phi)}{3 \sqrt{2} \eta r^{2}},
\end{aligned}
$$

$$
\begin{aligned}
& \left(110\left|\overline{\boldsymbol{\mu}}_{12}\right| 110\right)=\frac{1}{2 \eta r}-\frac{0.282566(2 a)^{2}}{\eta r^{3}}, \\
& \left(1-10\left|\overline{\boldsymbol{\mu}}_{12}\right| 110\right)=-\frac{1}{6 \eta r}+\frac{0.232928(2 a)^{2} \exp (2 i \phi)}{\eta r^{3}} .
\end{aligned}
$$

The remaining multipole elements of $\overline{\boldsymbol{\mu}}_{12}$ are obtained from the symmetry properties,

$\left(1 m^{\prime} \sigma^{\prime}\left|\overline{\boldsymbol{\mu}}_{12}\right| 1 m \sigma\right)=(-1)^{\sigma+\sigma^{\prime}}\left(1 m \sigma\left|\overline{\boldsymbol{\mu}}_{12}\right| 1 m^{\prime} \sigma^{\prime}\right)^{*}$, 


$$
\begin{aligned}
& \left(1-m^{\prime} \sigma^{\prime}\left|\overline{\boldsymbol{\mu}}_{12}\right| 1-m \sigma\right) \\
& \quad=(-1)^{\sigma+\sigma^{\prime}+m+m^{\prime}}\left(1 m^{\prime} \sigma^{\prime}\left|\overline{\boldsymbol{\mu}}_{12}\right| 1 m \sigma\right)^{*},
\end{aligned}
$$

where $\left(l^{\prime} m^{\prime} \sigma^{\prime}\right),(l m \sigma) \in\{(110),(1-10),(101)\}$.

To construct the Cartesian form of the operators $\overline{\boldsymbol{\mu}}_{11}$ and $\overline{\boldsymbol{\mu}}_{12}$, we follow the scheme presented in Ref. 50 . The idea is to transform from the complex multipole functions to their real linear combinations. This will eventually lead to the vector Cartesian components of the forces, torques, and velocities. The coefficients of these transformations form the elements of a unitary matrix denoted as $\mathbf{X}$ in Ref. 50 (note that there is a misprint in Eq. (B6) of Ref. 50, i.e., $\mathbf{X}$ should be replaced by $\mathbf{X}^{\dagger}$ and vice versa). In the quasi-two-dimensional case, the corresponding matrix elements $\mathbf{X}\left(1 m^{\prime} \sigma^{\prime}, 1 m \sigma\right)$ are labeled by $\left(m^{\prime}, \sigma^{\prime}\right),(m, \sigma) \in\{(10),(-10),(01)\}$ only. Explicitly

$$
\mathbf{X}=\frac{1}{\sqrt{2}}\left(\begin{array}{ccc}
1 & i & 0 \\
-1 & i & 0 \\
0 & 0 & i \sqrt{2}
\end{array}\right)
$$

Using Eqs. (B7) and (B8) from Ref. 50, we obtain the following transformation from the spherical to the Cartesian form of the operators $\overline{\boldsymbol{\mu}}_{1 k}$, with $k=1,2$,

$$
\begin{aligned}
\overline{\boldsymbol{\mu}}_{1 k}= & \sum \frac{3}{4 \pi}(-1)^{m^{\prime}+m} X^{\dagger}\left(m^{\prime} \sigma^{\prime}, m^{\prime \prime} \sigma^{\prime \prime}\right) \\
& \times\left(1 m^{\prime \prime} \sigma^{\prime \prime}\left|\overline{\boldsymbol{\mu}}_{1 k}\right| 1 m^{\prime \prime \prime} \sigma^{\prime \prime \prime}\right) X\left(m^{\prime \prime \prime} \sigma^{\prime \prime \prime}, m \sigma\right),
\end{aligned}
$$

where the sum is taken over $\left(m^{\prime \prime} \sigma^{\prime \prime}\right),\left(m^{\prime \prime \prime} \sigma^{\prime \prime \prime}\right) \in\{(1,0)$, $(-1,0),(0,1)\}$.

${ }^{1}$ S. Kim and S. J. Karrila, Microhydrodynamics: Principles and Selected Applications (Butterworth, London, 1991).

${ }^{2}$ L. Durlofsky, J. F. Brady, and G. Bossis, J. Fluid Mech. 180, 21 (1987).

${ }^{3}$ B. Cichocki, B. U. Felderhof, K. Hinsen, E. Wajnryb, and J. Bławzdziewicz, J. Chem. Phys. 100, 3780 (1994).

${ }^{4}$ P. N. Pusey, in Liquids, Freezing and Glass Transitions, edited by J. P. Hansen, D. Levesque, and J. Zinn-Justin (North-Holland, Amsterdam, 1991).

${ }^{5}$ R. B. Jones and P. N. Pusey, Annu. Rev. Phys. Chem. 42, 137 (1991).

${ }^{6}$ J. K. G. Dhont, An Introduction to Dynamics of Colloids (Elsevier, Amsterdam, 1996).

${ }^{7}$ W. Russel, D. A. Saville, and W. R. Schowalter, Colloidal Dispersions (Cambridge University Press, Cambridge, 1989).

${ }^{8}$ G. Nägele, Phys. Rep. 272, 215 (1996).

${ }^{9}$ J. Blake, Proc. Cambridge Philos. Soc. 70, 303 (1971).

${ }^{10}$ K. Aderogba and J. R. Blake, Bull. Aust. Math. Soc. 18, 345 (1978).

${ }^{11}$ C. W. J. Beenakker and P. Mazur, Phys. Fluids 28, 767 (1985).

${ }^{12}$ G. Bossis and A. Meunier, Phys. Fluids A 3, 1853 (1991).

${ }^{13}$ R. B. Jones and B. Cichocki, Physica A 258, 273 (1998).

${ }^{14}$ R. Jones and R. Kutteh, J. Chem. Phys. 112, 11080 (2000).

${ }^{15}$ R. B. Jones, B. U. Felderhof, and J. M. Deutch, Macromolecules 8, 680 (1975).

${ }^{16}$ S. H. Lee, R. S. Chadwick, and G. Leal, J. Fluid Mech. 93, 705 (1979).

${ }^{17}$ J. Bławzdziewicz, V. Cristini, and M. Loewenberg, Phys. Fluids 11, 251 (1999).
${ }^{18}$ L. Lobry and N. Ostrowsky, Phys. Rev. B 53, 12050 (1996).

${ }^{19}$ M. D. Carbajal-Tinoco, F. Castro-Román, and J. L. Arauz-Lara, Phys. Rev. E 53, 3745 (1996).

${ }^{20}$ M. D. Carbajal-Tinoco, F. Cruz de León, and J. L. Arauz-Lara, Phys. Rev. E 56, 6962 (1997).

${ }^{21}$ H. Acuna-Campa, M. D. Carbajal-Tinoco, J. L. Arauz-Lara, and M. Medina-Noyola, Phys. Rev. Lett. 80, 5802 (1998).

${ }^{22}$ J. Santana-Solano and J. L. Arauz-Lara, Phys. Rev. E 65, 021406 (2002).

${ }^{23}$ A. H. Marcus, J. Schofield, and S. A. Rice, Phys. Rev. E 60, 5725 (1999).

${ }^{24}$ R. Pesché and G. Nägele, Phys. Rev. E 62, 5432 (2000).

${ }^{25}$ R. Pesché and G. Nägele, Phys. Rev. E 60, 5725 (1999).

${ }^{26}$ R. Pesché and G. Nägele, Europhys. Lett. 51, 584 (2000).

${ }^{27}$ S. Bhattacharya and J. Bławzdziewicz, J. Math. Phys. 43, 5720 (2002).

${ }^{28}$ S. H. Lee and L. G. Leal, J. Fluid Mech. 98, 193 (1980).

${ }^{29}$ B. Lin, S. A. Rice, and D. A. Weitz, J. Chem. Phys. 99, 8308 (1993).

${ }^{30}$ B. Lin, S. A. Rice, and D. A. Weitz, Phys. Rev. E 51, 423 (1995).

${ }^{31}$ K. Zahn, J. M. Mendez-Alcaraz, and G. Maret, Phys. Rev. Lett. 79, 175 (1997).

${ }^{32}$ B. Rinn, K. Zahn, P. Maass, and G. Maret, Europhys. Lett. 46, 537 (1999).

${ }^{33}$ K. Zahn and G. Maret, Phys. Rev. Lett. 85, 3656 (2000).

${ }^{34}$ R. Pesché, M. Kollmann, and G. Nägele, J. Chem. Phys. 114, 8701 (2001).

${ }^{35}$ G. Nägele, M. Kollmann, R. Pesché, and A. Banchio, Mol. Phys. 100, 2921 (2002)

${ }^{36}$ M. Kollmann, R. Hund, B. Rinn et al., Europhys. Lett. 58, 919 (2002).

${ }^{37}$ A. E. Larsen and D. G. Grier, Nature (London) 385, 230 (1997).

${ }^{38}$ T. M. Squires and M. P. Brenner, Phys. Rev. Lett. 85, 4976 (2000).

${ }^{39}$ E. R. Dufresne, T. M. Squires, M. P. Brenner, and D. G. Grier, Phys. Rev. Lett. 85, 3317 (2000).

${ }^{40}$ M. L. Kurnaz and J. V. Maher, Phys. Rev. E 53, 978 (1996).

${ }^{41}$ I. Pagonabarraga and J. M. Rubi, Phys. Rev. Lett. 73, 114 (1994).

${ }^{42}$ K. Zahn, R. Lenke, and G. Maret, Phys. Rev. Lett. 82, 2721 (1999).

${ }^{43}$ P. Pieranski, Phys. Rev. Lett. 45, 569 (1980).

${ }^{44}$ K. Zahn, Ph.D. thesis Université Louis Pasteur de Strasbourg, 1997.

${ }^{45} \mathrm{~J}$. Rowlinson and B. Widom, Molecular Theory of Capillarity (Clarendon, Oxford, 1982).

${ }^{46}$ S. Safran, Statistical Thermodynamics of Surfaces, Interfaces, and Membranes (Addison-Wesley, New York, 1994).

${ }^{47}$ H. Princen, Surface and Colloid Science (Wiley-Interscience, New York 1969), Vol. 2.

${ }^{48}$ S. Lee and L. Leal, J. Colloid Interface Sci. 87, 81 (1982).

${ }^{49}$ S. Lee, R. Chadwick, and L. Leal, J. Fluid Mech. 93, 705 (1979).

${ }^{50}$ B. Cichocki, R. B. Jones, R. Kutteh, and E. Wajnryb, J. Chem. Phys. 112, 2548 (2000).

${ }^{51}$ R. G. Cox and H. Brenner, J. Fluid Mech. 28, 391 (1967).

${ }^{52}$ P. Mazur and D. Bedeaux, Physica (Amsterdam) 76, 235 (1974).

${ }^{53}$ B. U. Felderhof, Physica A 84, 557 (1976).

${ }^{54}$ B. U. Felderhof, Physica A 84, 569 (1976).

${ }^{55}$ R. Schmitz and B. U. Felderhof, Physica A 92, 123 (1978).

${ }^{56}$ B. U. Felderhof, Physica A 151, 1 (1988).

${ }^{57}$ D. J. Jeffrey and Y. Onishi, J. Fluid Mech. 139, 261 (1984).

${ }^{58}$ C. Pozrikidis, Boundary Integral and Singularity Methods for Linearized Viscous Flow (Cambridge University Press, Cambridge, 1992).

${ }^{59}$ R. Schmitz and B. U. Felderhof, Physica A 113, 90 (1982).

${ }^{60}$ H. Lamb, Hydrodynamics (Dover, New York, 1945).

${ }^{61}$ B. U. Felderhof and R. B. Jones, Physica A 93, 457 (1978).

${ }^{62}$ B. U. Felderhof and R. B. Jones, J. Math. Phys. 30, 339 (1989).

${ }^{63}$ B. U. Felderhof, Physica A 89, 373 (1977).

${ }^{64}$ R. Schmitz and B. U. Felderhof, Physica A 116, 163 (1982).

${ }^{65}$ G. Perkins and R. B. Jones, Physica A 171, 575 (1991).

${ }^{66}$ B. Cichocki, B. U. Felderhof, and R. Schmitz, PCH, PhysicoChem. Hydrodyn. 10, 383 (1988).

${ }^{67}$ B. Cichocki, M. L. Ekiel-Jeżewska, and E. Wajnryb (unpublished).

${ }^{68}$ J. Rotne and S. Prager, J. Chem. Phys. 50, 4831 (1969). 